\title{
Konzeption
}

\section{Die Konzeption dieses Buchs}

Dieses Buch dient einerseits dem Studium begleitend zu einer arbeitsrechtlichen Grundvorlesung, insbesondere dann, wenn keine begleitende Arbeitsgemeinschaft angeboten wird. Andererseits soll es auch dem effizienten Repetieren und der Vorbereitung auf das Examen dienen. Die Lösungsvorschläge sind ausformuliert und konzentrieren sich auf das, was von Prüfungsarbeiten im Pflichtfach noch erwartet werden könnte. Probleme werden weniger anhand einer klausuruntypischen Auseinandersetzung mit Rechtsprechung und Literatur der vergangenen Jahrzehnte, sondern vor allem durch normativ angebundene Argumente anhand der Auslegungs- und Rechtsfortbildungsmethoden entfaltet. Denn in Klausuren des Pflichtfachs Arbeitsrecht geht es erfrischenderweise weniger um die Reproduktion fremder Methode, sondern um die Kenntnis von Grundstrukturen bei Problembewusstsein im Übrigen. Die Nachweise sind bewusst reduziert, am Ende jedes Falls finden sich aber konkrete Hinweise auf die entsprechenden Stellen jeweils in einem kurzen und einem langen Lehrbuch. Über das, was man im Gutachten schreiben sollte und könnte hinausgehende Hinweise sind abgesetzt in Kästen dargestellt. Aus didaktischen Gründen sind die Lösungsvorschläge aber meist ausführlicher als das, was in der Klausursituation geleistet werden könnte. Zusammengenommen können die Kästen als grundlegendes Repetitorium verwendet werden. Im Wesentlichen entsprechen sie dem, was in einem kompakten arbeitsrechtlichen Repetitorium gelehrt werden könnte. Inhaltlich decken die Fälle den Kern der prüfungsrelevanten Materie ab. Die 21 Fälle teilen sich daher auf in:

1. Fälle 1-8 zum Erlernen und Wiederholen der Grundlagen. Kernfragen des Individualarbeitsrechts sind (1) Arbeitnehmerbegriff, (2) Mängel des Arbeitsvertrags, (3) AGB-Kontrolle des Arbeitsvertrags, (4) Gleichbehandlung im Arbeitsverhältnis, (5) Lohn ohne Arbeit, (6) Besonderheiten der Haftung, (7) Kündigung und (8) Kündigungsschutzklage. Die Fälle hier sind Einführungsfälle mit wenigen Problemen und einer spezifischen Frage. Die erläuternden Kästen befassen sich mit Kernaspekten des Stoffs.

2. Fälle 9-15 sind Fälle, die eine Semesterabschlussklausur der Grundvorlesung Arbeitsrecht (oder auch ein größerer AG-Fall) sein könnten ${ }^{1}$ oder waren. Die Fälle hier zeichnet aus, dass sie Probleme aus mehreren Teilbereichen des Individualarbeitsrechts schon komplexerer Art enthalten und dass eine Bearbeitung unter Klausurbedingungen in 120 Minuten erwartet würde. Würden diese Fälle mit Elementen anderer Semesterabschlussklausuren angereichert, hätten diese Fälle größtenteils schon Examensniveau. Die erläuternden Kästen befassen sich zum Teil mit einer Vertiefung des Stoffs, zum Teil mit alternativen Lösungsmöglichkeiten.

3. Fälle 16-21 sind Fälle, die Examensklausuren sein könnten oder waren. Die Fälle hier zeichnet die Vielzahl an Problemen aus mehreren Teilbereichen komplexerer Art und zum Teil eine Verzahnung mit Problemen des allgemeinen Zivilrechts oder des Zivilprozessrechts aus. Eine Bearbeitung würde in 5 Stunden erwartet werden, Ausschnitte wären auch als Se-

1 Es wurden nicht ausschließlich Original-Semesterabschlussklausuren verwendet, um innerhalb dieses Buchs eine vollständigere thematische Abdeckung zu erreichen. 
mesterabschlussklausur oder größerer AG-Fall geeignet. Die erläuternden Kästen und in kursiv gesetzten Bemerkungen befassen sich zu einem kleineren Teil mit einer Vertiefung des Stoffs, größtenteils mit alternativen Lösungsmöglichkeiten und klausurtaktischen Hinweisen.

Die Schemata und Übersichten am Ende des Buchs dienen dem schnellen Wiederholen des Stoffs. 